\title{
A finite element approach to $x$-ray optics design
}

\section{Honkanen, Ari-Pekka}

2017-05-15

Honkanen, A-P , Ferrero , C , Guigay , J-P \& Mocella , V 2017 , ' A finite element approach to $x$-ray optics design ' , Proceedings of SPIE, the International Society for Optical Engineering , vol. 10236 . https://doi.org/10.1117/12.2268072

http://hdl.handle.net/10138/214442

https://doi.org/10.1117/12.2268072

submittedVersion

Downloaded from Helda, University of Helsinki institutional repository.

This is an electronic reprint of the original article.

This reprint may differ from the original in pagination and typographic detail.

Please cite the original version. 


\title{
A Finite Element Approach to X-ray Optics Design
}

\author{
A.P. Honkanen ${ }^{\mathrm{a}}$, C. Ferrero ${ }^{\mathrm{b}}$, J.P. Guigay ${ }^{\mathrm{b}}, \&$ V. Mocella ${ }^{\mathrm{c}}$
}

a Department of Physics, PO Box 64, FI-00014 Helsinki, Finland

b ESRF-The European Synchrotron Radiation Facility, Grenoble, France

c CNR-IMM Consiglio Nazionale delle Ricerca- Istituto per la Microelettronica e Microsistemi, Naples, Italy

\section{Introduction}

Dynamical diffraction in a deformed (often bent) crystal is described by the Takagi equations ${ }^{1}$ which, in general, have to be solved numerically on a regular 2-D grid of points representing a planar cross section of the crystal in which the diffraction of an incident X-ray wavefront occurs . Presently, the majority of numerical approaches are based on a finite difference solving scheme ${ }^{2-4}$ which can be easily implemented on a regular Cartesian grid but is not suitable for deformed meshes. In this case, the inner deformed crystal structure can be taken into account, but not the shape of the crystal surface if this differs substantially from a planar profile ${ }^{5,6}$.

Conversely, a finite element method (FEM) can be easily applied to a deformed mesh and serves very well to the purpose of modelling any incident wave on an arbitrarily shaped entrance surface ${ }^{7}$ e.g. that of a bent crystal or a crystal submitted to a strong heat load ${ }^{8-10}$.

For instance, the cylindrical shape of the surface of a strongly bent crystal plate can easily be taken into account in a FEM calculation. Bent crystals are often used as focusing optical elements in Xray beamlines ${ }^{11-13}$.

In the following, we show the implementation of a general numerical framework for describing the propagation of X-rays inside a crystal based on the solution of the Takagi equations via the COMSOL Multiphysics FEM software package (www.comsol.com). A cylindrically bent crystal will be taken as an example to illustrate the capabilities of the new approach.

\section{Theoretical frame}

Considering an incident wave $\Psi_{i n c}=\exp (i \vec{k} \cdot \vec{r}) E_{i n c}(\vec{r})$ and the corresponding wave-field in a deformed crystal as $\psi(\vec{r})=\exp (i \vec{k} \cdot \vec{r})\left[E_{o}(\vec{r})+\exp (i \vec{h} \cdot \vec{r}) E_{h}(\vec{r})\right]$ where the modulation amplitudes are slowly varying functions, it can be shown that the modified amplitudes

$$
\begin{aligned}
& T\left(s_{o}, s_{h}\right)=\exp \left[i \frac{k}{2} \chi_{o}\left(s_{o}+s_{h}\right)-i k s_{h} \Delta \theta \sin 2 \theta_{B}\right] E_{o}\left(s_{o}, s_{h}\right) \\
& D\left(s_{o}, s_{h}\right)=\exp \left[i \frac{k}{2} \chi_{o}\left(s_{o}+s_{h}\right)-i k s_{h} \Delta \theta \sin 2 \theta_{B}-i \vec{h} \cdot \vec{u}\right] E_{h}\left(s_{o}, s_{h}\right)
\end{aligned}
$$


are solutions of the following Takagi equations

$$
\begin{aligned}
& \frac{\partial T}{\partial s_{0}}=i \frac{k}{2} \chi_{\bar{h}} D \\
& \frac{\partial D}{\partial s_{h}}=-i \frac{\partial(\vec{h} \cdot \vec{u})}{\partial s_{h}} D+i \frac{k}{2} \chi_{h} T
\end{aligned}
$$

In the above equations, $\left(s_{o}, s_{h}\right)$ are oblique coordinates along the directions of $\vec{k}$ and $\vec{k}_{h}=\vec{k}+\vec{h}$, respectively; $\vec{u}(\vec{r})$ is the deformation field, $\chi_{o, h, \bar{h}}$ are the relevant Fourier coefficients of the undeformed crystal polarizability (to be multiplied by $\cos 2 \theta_{B}$ in the case of $\pi$ instead of $\sigma$ polarization); $\theta_{B}$ is the Bragg angle and $\Delta \theta$ is the crystal misorientation from the geometrical Bragg position.

In order to numerically solve the system of equations $(2 \mathrm{a}, 2 \mathrm{~b})$ we present a new Finite Element Method (FEM) scheme based on the following integral weak formulation of these equations:

$$
\begin{aligned}
& \iint_{\Omega} d \Omega\left[\frac{\partial \omega_{0}}{\partial s_{o}} T+i \frac{k}{2} \chi_{-h} \omega_{i} D\right]-\int_{\Gamma} d l \vec{n} \cdot \hat{s}_{o} \omega_{i} T=0 \\
& \iint_{\Omega} d \Omega\left[\frac{\partial \omega_{i}}{\partial s_{h}} D+i \frac{k}{2} \chi_{h} T+i \frac{\partial(\vec{h} \cdot \vec{u})}{\partial s_{h}} D\right]-\int_{\Gamma} d l \vec{n} \cdot \hat{s}_{h} \omega_{i} D=0
\end{aligned}
$$

where $\omega_{i}(\vec{r})$ is a set of test functions; $\hat{s}_{o, h}$ are unit vectors in the $s_{o, h}$ directions. The FEM code generates a mesh of 2-D elements (generally triangles) adapted to the geometry of the integration domain $\Omega$ with boundary $\Gamma$. The boundary conditions are included in the boundary line integrals. In the chosen discretization scheme, a linear shape function $N_{i}(\vec{r})$ is associated to each knot $(i)$ of the mesh, ranging from the value 1 at the knot $(i)$ to 0 on the polygonal line formed by the other knots that are apices of the triangles sharing $(i)$ as apex. Test functions analogous to the chosen shape functions can be used. An approximate solution in the form $T(\vec{r})=\sum_{i} T_{i} N_{i}(\vec{r})$ and $D(\vec{r})=\sum_{i} D_{i} N_{i}(\vec{r})$, with coefficients $T_{i}$ and $D_{i}$ to be numerically determined, is calculated. From this solution, it is in general necessary to use the relations $(1 \mathrm{a}, 1 \mathrm{~b})$ to obtain the physically significant amplitudes $E_{o, h}(\vec{r})$. This is essential for the simulation of the focusing effect considered hereafter. 


\section{Numerical simulations}

We present FEM simulations of reflectivity curves of a flat undeformed crystal (Figs. 1 and 2) and of a cylindrically bent crystal (Fig. 3), both in symmetric Bragg geometry, as well as the related focusing properties, as implemented by using COMSOL. We consider a monochromatic point source in all cases. The Cartesian components of the displacement field, with the $z$-axis along the inward normal to the plate surface, are $U_{x}=x(z-t / 2) / R$ and $U_{z}=-\left[x^{2}+v(z-t / 2)^{2}\right] / 2 R$, where

$R$ is the bending radius, $t$ is the plate thickness and the Poisson ratio was chosen to be $v=0.27$.

The source-to-crystal distance $p$ is varied by using $E_{\text {inc }}(x)=\exp \left[i k\left(x \sin \theta_{B}\right)^{2} / 2 p-x^{2} / w^{2}\right]$ on the crystal surface. The curved shape of the surface of the bent crystal is taken into account in the FEM simulation: we have verified that the results do not change significantly if this surface is considered to be flat.

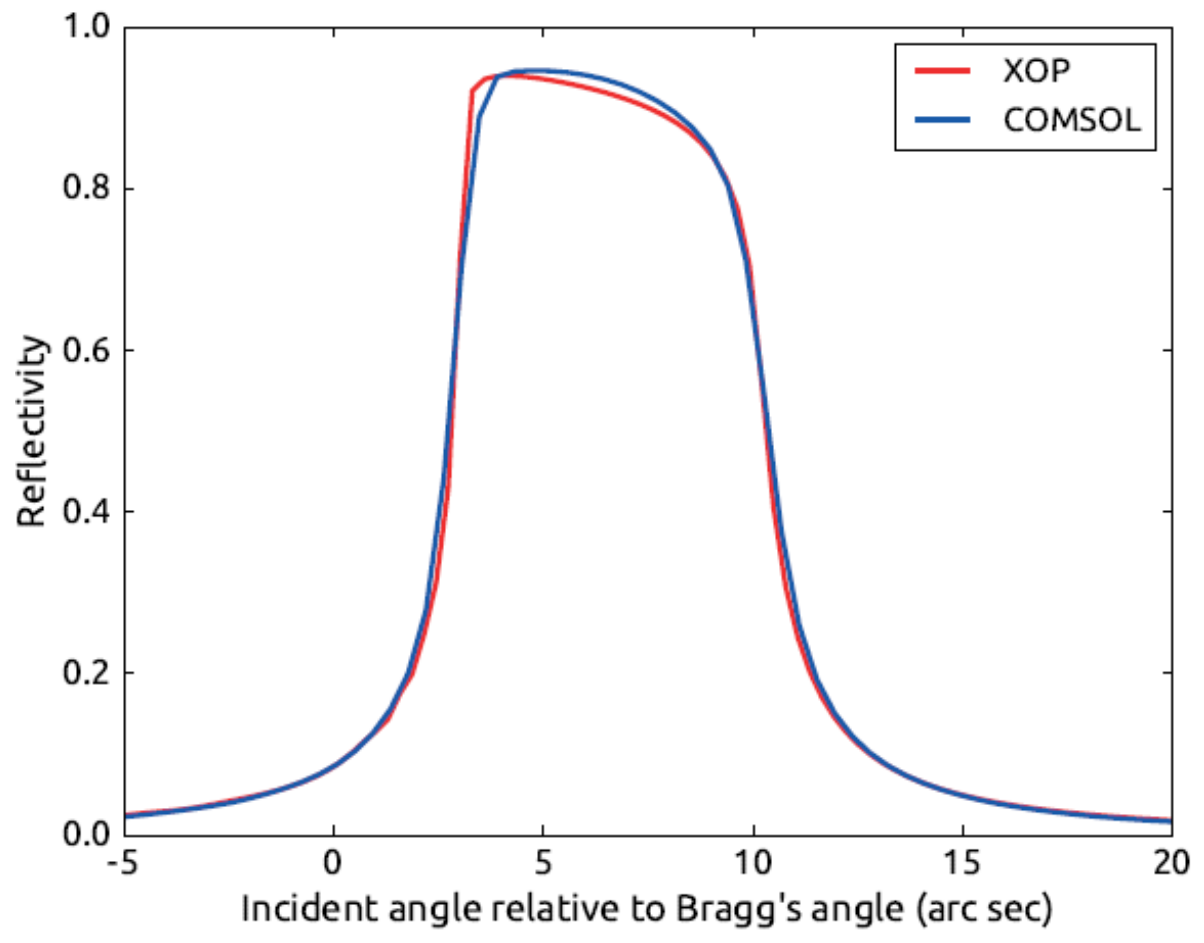

Fig.1 FEM simulation, using a triangular grid size of $1 \mu \mathrm{m}$, of the (Si111, E=8 Kev) reflectivity curve $(\mathrm{RC})$ of a $100 \mu \mathrm{m}$ thick undeformed crystal plate in symmetric Bragg geometry, compared to 
the output of the XOP INPRO program taken as a reference ${ }^{14}$. In the COMSOL simulation, the footprint of the incident plane wave on the crystal surface is modulated by a Gaussian window $(\sigma=$ $w / \sqrt{\operatorname{Ln} 2}=100 \mu \mathrm{m})$ implying the boundary condition:

$T(x, z=0)=\exp \left[i k x\left(\Delta \theta \sin 2 \theta_{B}+\chi_{o} / 2 \cos \theta_{B}\right)-x^{2} / w^{2}\right]$ on the crystal surface.

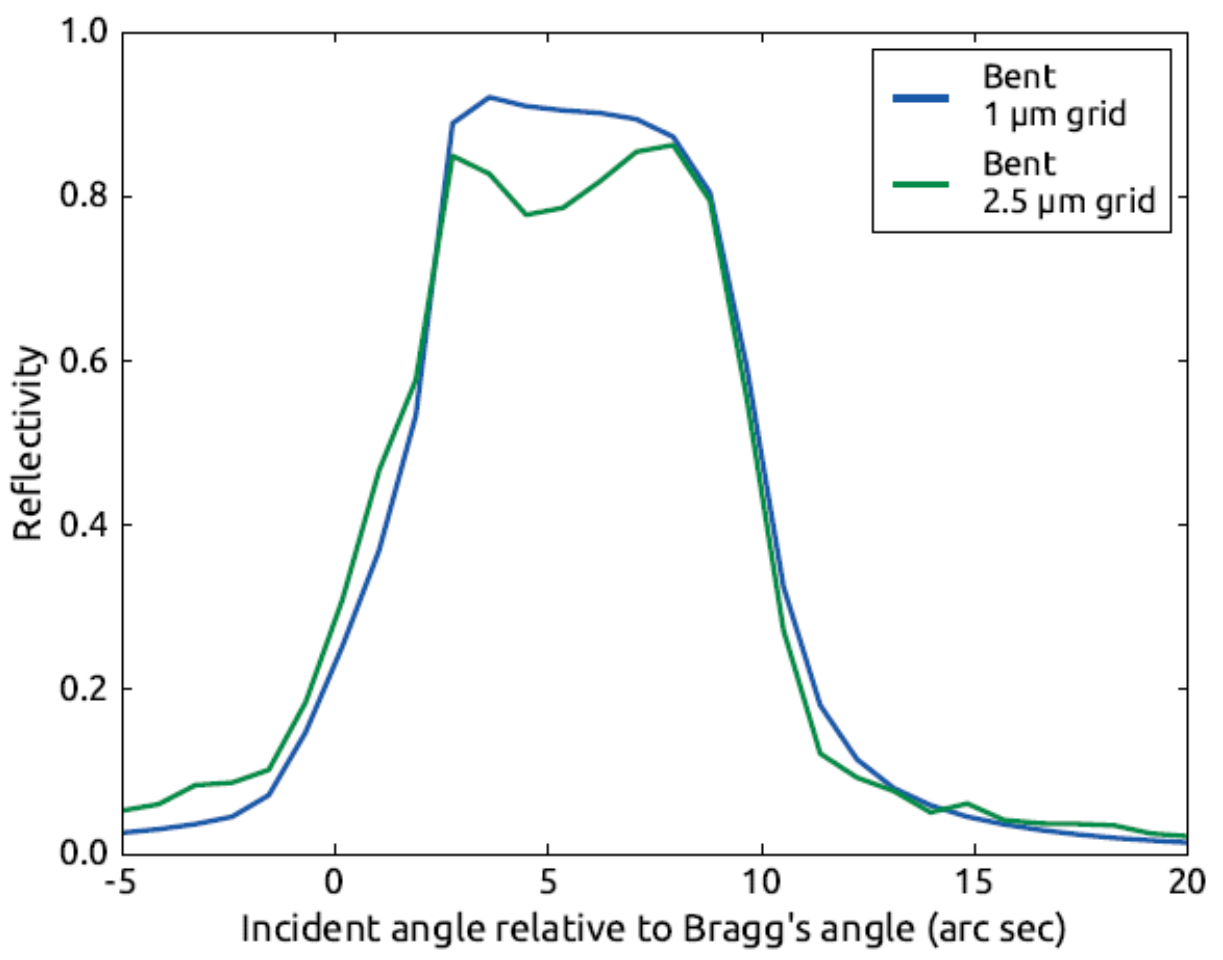

Fig2. RC simulation as in fig.1, but with two different grid sizes, showing that a grid size as small as $1 \mu \mathrm{m}$ is necessary to obtain reliable results; the accuracy can also be improved by using a "boundary layer option" available in COMSOL. 


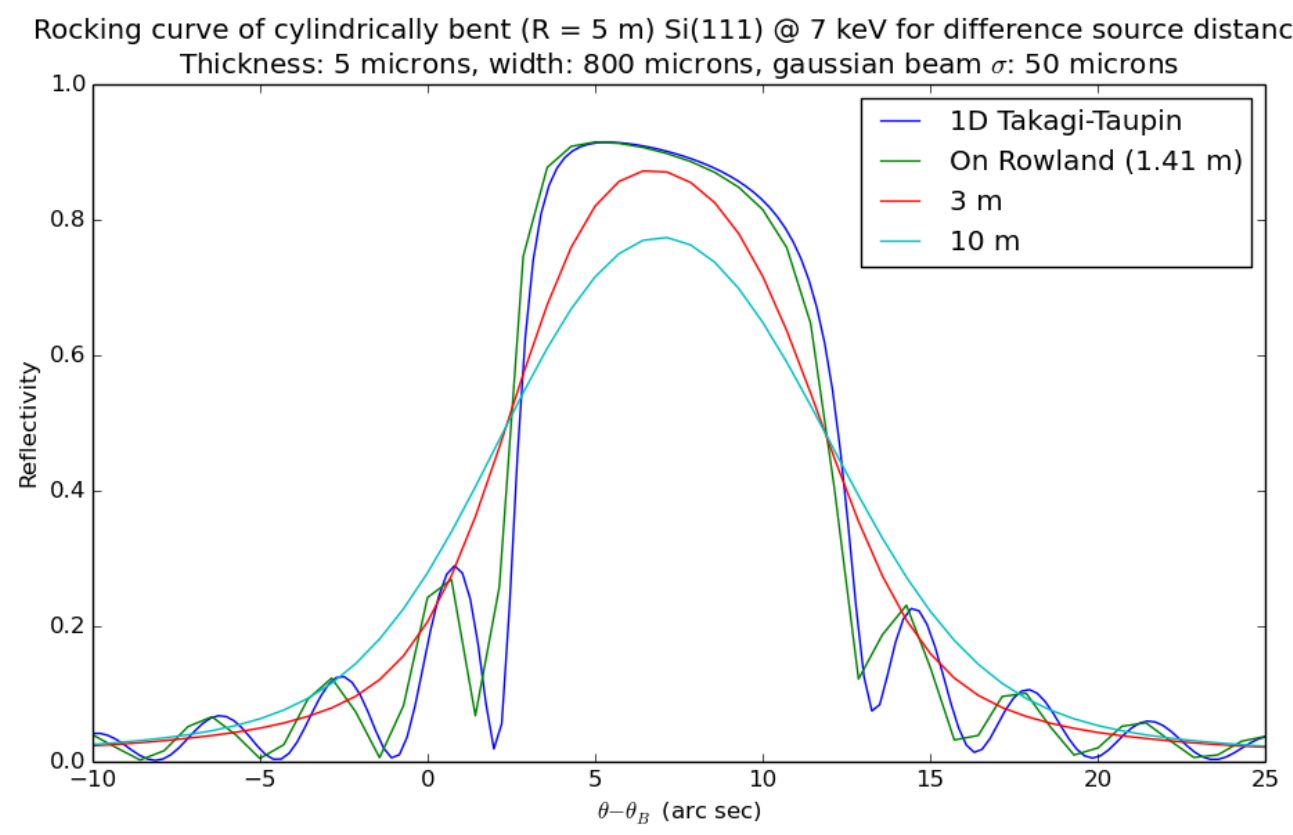

Fig. 3. RC simulations of the (Si111, $7 \mathrm{Kev)} \mathrm{of} 5 \mu \mathrm{m}$ thick cylindrically bent crystal plate (bending radius $R=5 \mathrm{~m}$ ) in symmetric Bragg geometry for different source-to-crystal distances. The extinction length $(0.735 \mu \mathrm{m})$ in this case is much smaller than the plate thickness. The other parameters are reported in the title of the Figs. $4 \mathrm{a}$ and $4 \mathrm{~b}$ display the maps of the total intensities inside a bent crystal $(R=5 \mathrm{~m})$ for two cases: a) source on the Rowland circle (Si111, $\mathrm{E}=8 \mathrm{Kev}$; $\left.p=R \sin \theta_{B}=1.40 \mathrm{~m}\right)$. b) $p=5 \mathrm{~m}$.

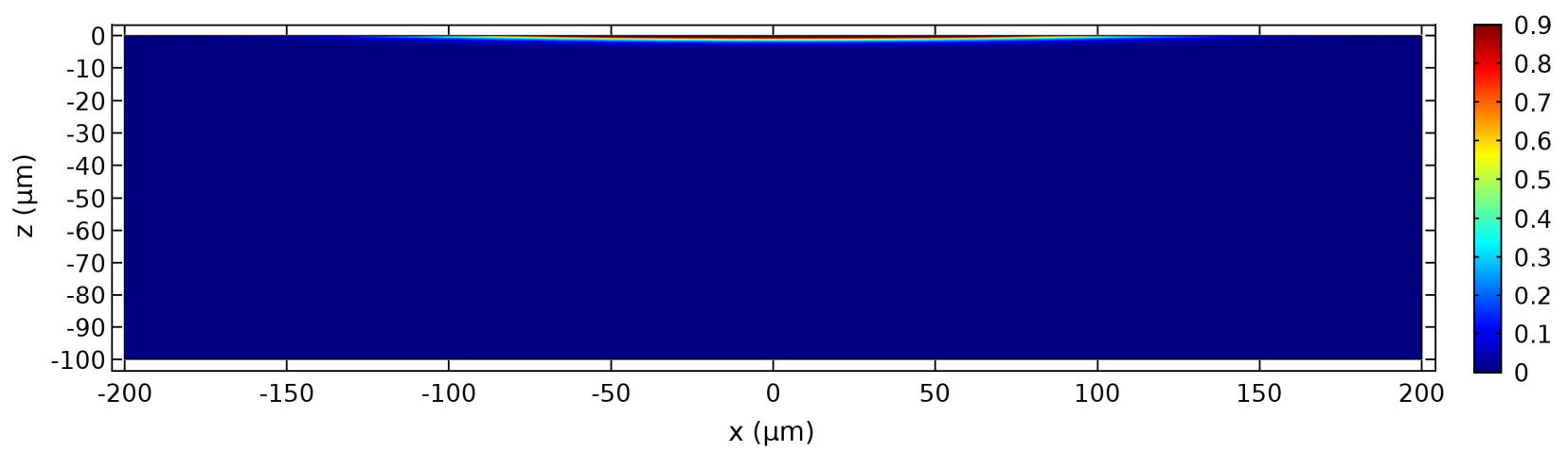

Fig. 4a. Map of the total intensity in the crystal illuminated by a source on the Rowland circle. Same parameters as in Fig.1. The beam penetration is limited to a very slight crystal depth 


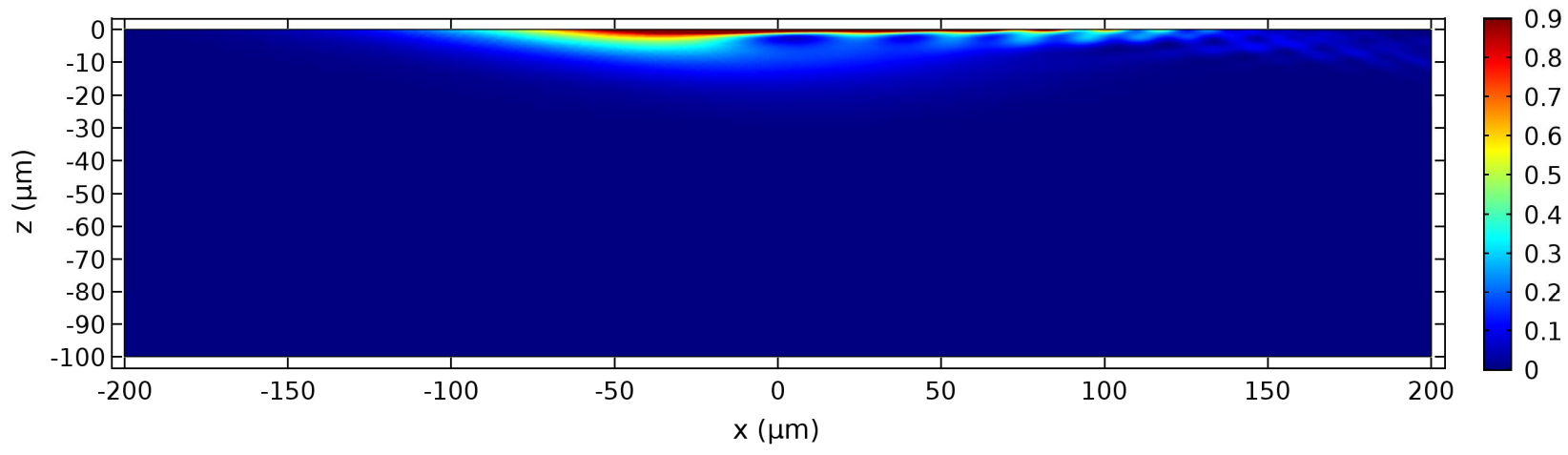

Fig. 4b. Map of the total intensity in the crystal illuminated by a source off-Rowland circle. To be noticed: the "mirage effect"

Fig. 5 shows the reflected intensity distributions on a bent crystal surface $(R=5 \mathrm{~m})$, in the case of the same source at different distances; the Gaussian window $(\sigma=200 \mu \mathrm{m})$ is centered at the crystal surface point corresponding to the center of the RC. The reflected intensity distribution is not very different from the incident one if the source is on the Rowland circle $(p=1.236 \mathrm{~m})$, indicating almost total reflection as expected, with, accordingly, a small penetration range in the crystal, as shown in fig. 4a. This is no longer the case for $p=5 \mathrm{~m}$ : in this case a part of the incident wave penetrates in the crystal and is reflected back towards the surface due to the "mirage effect" 15,16 . This gives rise by interference to the oscillations seen in Fig.5. By using (1b), the reflected amplitude $E_{h}\left(s_{o}, s_{h}\right)$ is obtained from the reflected amplitude $D\left(s_{o}, s_{h}\right)$ provided by the solution of (2a, 2b). The distribution of $E_{h}\left(s_{o}, s_{h}\right)$ on the crystal surface can be projected on a plane perpendicular to the reflected direction and further propagated in free space, in order to investigate its focusing properties. The focusing distance is found to be in agreement with the lens equation $1 / p+1 / q=2 / R \sin \theta_{B}{ }^{17}$. The corresponding intensity distributions in the focus position is plotted in fig.6, for the source-to-crystal distances considered in fig.5. 


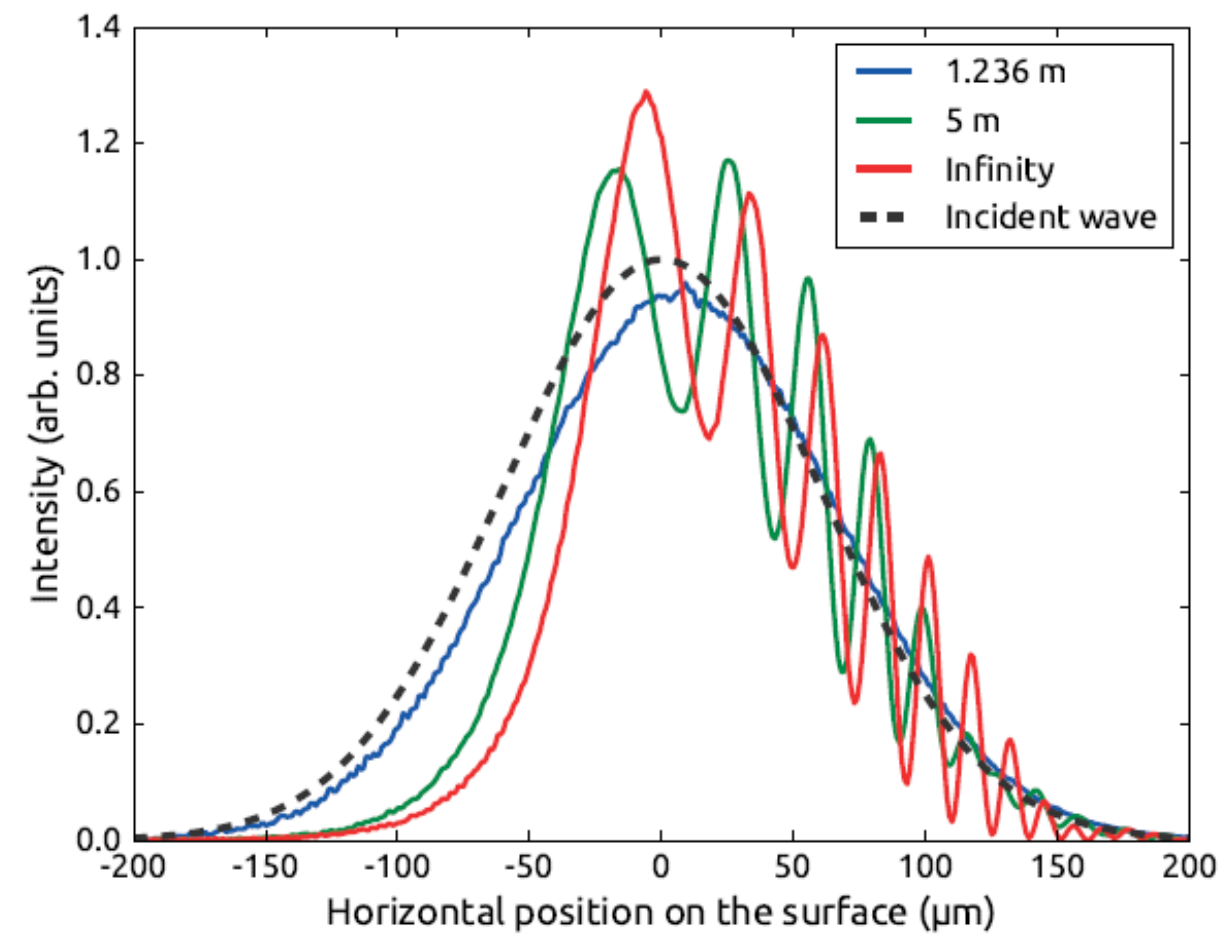

Fig. 5. Reflected intensity distributions on the crystal surface for different source-to-crystal distances compared with the incident wave intensity. 


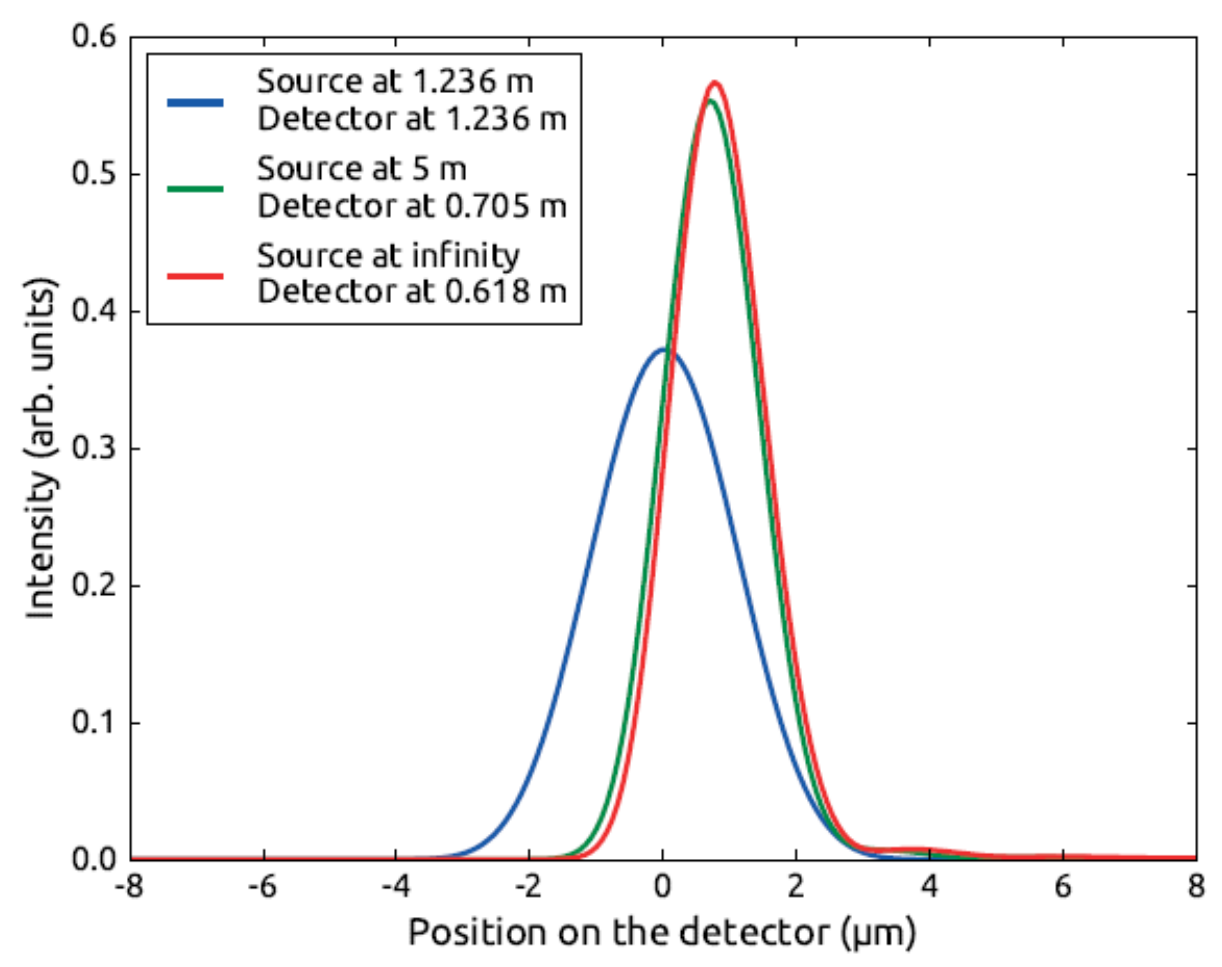

Fig.6. Focus intensity profiles in the same conditions as in fig.5

Figs. 7 and 8 show surface intensity distributions for two values of the misorientation angle. Because of the refraction effect, $\Delta \theta=0$ arcsec corresponds actually to an off-Bragg position; while $\Delta \theta=5$ arcsec corresponds to the effective Bragg condition. In Fig. 8, the intensity oscillations are due to the part of the beam reflected by the crystal back surface. The crystal thickness is $20 \mu \mathrm{m}$. 


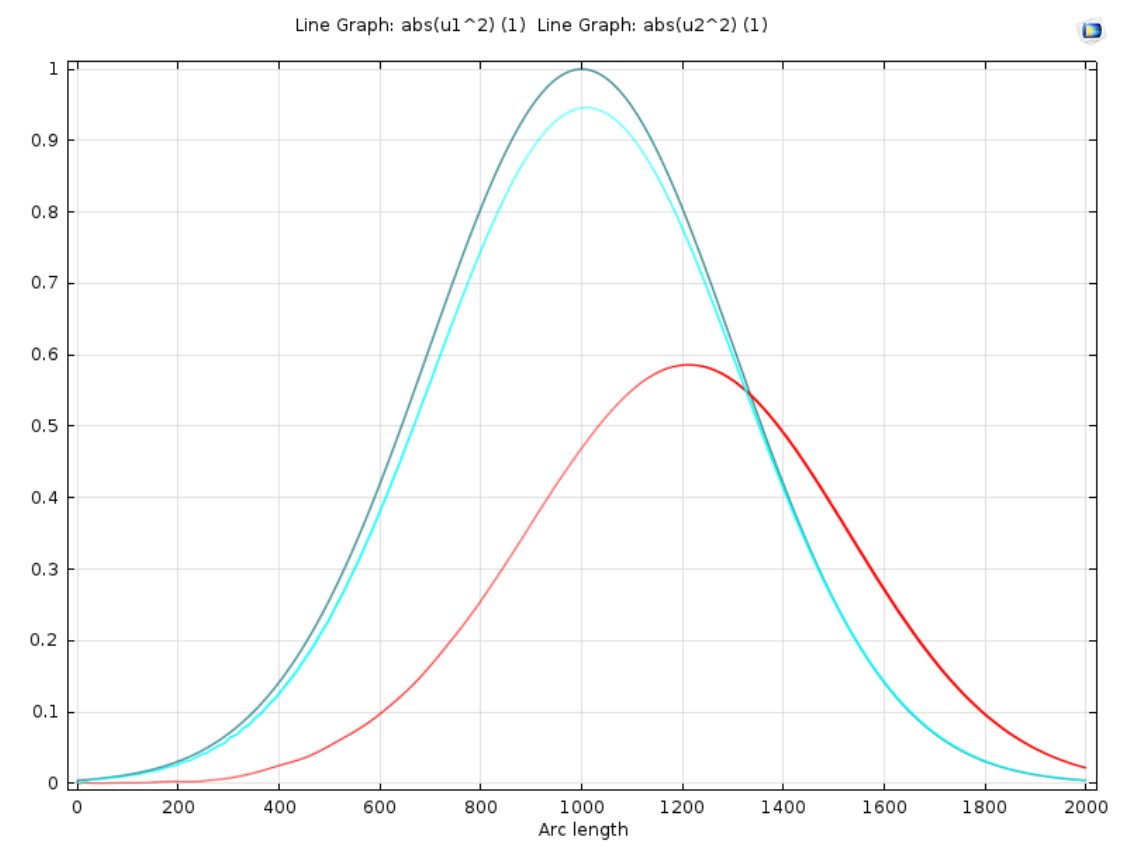

Fig. 7. Reflected intensity distributions on the crystal surface. Bending radius $10 \mathrm{~m}$; source on Rowland circle; Si 111, $12 \mathrm{Kev}$; red curve for $\Delta \theta=0$, green curve for $\Delta \theta=5 \operatorname{arcsec}$; the blue curve represents the incident intensity.

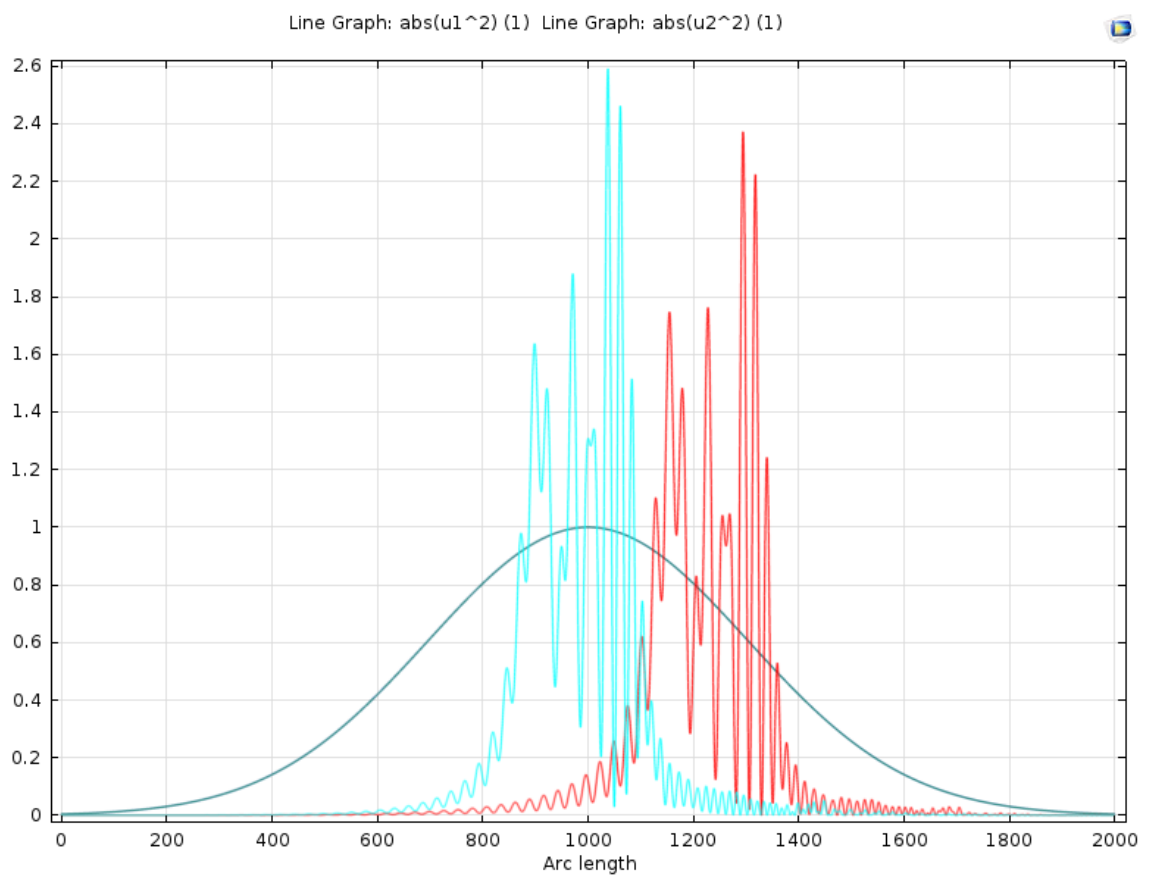

Fig. 8: Same conditions as in Fig. 7, except that the source is at a large distance $(\mathrm{p}=30 \mathrm{~m})$. 


\section{Conclusions}

A very general finite element approach to Takagi equations in their integral form is presented. This allows for wide flexibility in numerically simulating the X-ray propagation in both a flat and deformed crystal for any complex geometric domain. The finite element solution is computationally efficient and comparable to the finite difference method in conventional cases, where the computational domain is rectangular or, more broadly, delimited by straight lines. However, in general the deformation modifies this simple geometry and when the diffracted phase plays a crucial role, the deformed surface has to be accounted for in the calculations. In a forthcoming work we will demonstrate the phase propagation effects in air after diffraction from a crystal polychromator in Bragg geometry as simulated by our finite element code.

\section{References}

[1] Takagi, S., "A dynamical theory of diffraction for a distorted crystal," J Phys Soc Japan 26(5), 1239-1253 (1969).

[2] Epelboin, Y., "Simulation of X-ray topographs," Materials Science and Engineering 73, 143 (1985).

[3] Epelboin, Y., Mocella, V., Soyer, A., "Optical characteristics of synchrotron sources and their influence in the simulation of X-ray topographs," Philosophical Transactions of the Royal Society A: Mathematical, Physical and Engineering Sciences 357(1761), 2731-2739 (1999).

[4] Mocella, V., Lee, W. K., Tajiri, G., Mills, D., Ferrero, C., Epelboin, Y., “A new approach to the solution of the Takagi-Taupin equations for X-ray optics: application to a thermally deformed crystal monochromator," J Appl Crystallogr 36(1), 129-136 (2003).

[5] Honkanen, A. P., Monaco, G., Huotari, S., IUCr, "A computationally efficient method to solve the Takagi-Taupin equations for a large deformed crystal," J Appl Crystallogr 49(4), 1284-1289 (2016).

[6] Guigay, J. P., Morawe, C., Mocella, V., Ferrero, C., "An analytical approach to estimating aberrations in curved multilayer optics for hard x-rays: 1. Derivation of caustic shapes.," Opt. Express 16(16), 12050-12059 (2008).

[7] Mocella, V., Ferrero, C., Guigay, J. P., “ Dynamical diffraction approach of deformed crystals using finite element method," SPIE Optics+ .. 9510, R. Hudec and L. Pina, Eds., 95100H-95100H-6, International Society for Optics and Photonics (2015).

[8] Hoszowska, J., Migliore, J. S., Mocella, V., Ferrero, C., Freund, A. K., Zhang, L., "Performance of synchrotron X-ray monochromators under heat load Part 1: finite element modeling," Nuclear Instruments and Methods in Physics Research Section A: Accelerators, Spectrometers, Detectors and Associated Equipment 467-468, 409-413 (2001).

[9] Mocella, V., Ferrero, C., Freund, A. K., Hoszowska, J., "Performances of synchrotron X- 
ray monochromators under heat load. Part 2. Application of the Takagi-Taupin diffraction theory," Nuclear Instruments and ... 467-468, 414-417 (2001).

[10] Hoszowska, J., Mocella, V., Zhang, L., Migliore, J. S., Freund, A. K., Ferrero, C., "Performance of synchrotron X-ray monochromators under heat load.Part 3: Comparison between theory and experiment," Nuclear Instruments and Methods in Physics Research Section A: Accelerators, Spectrometers, Detectors and Associated Equipment 467-468, 631-634 (2001).

[11] Mocella, V., Guigay, J. P., Hrdy, J., Ferrero, C., Hoszowska, J., "Bent crystals in Laue geometry: dynamical focusing of a polychromatic incident beam," J Appl Crystallogr 37(6), 941-946 (2004).

[12] Lagomarsino, S., Cedola, A., Di Fonzo, S., Jark, W., "Advances in microdiffraction with xray waveguide," Crystal Research and Technology 37(7), 758-769 (2002).

[13] Di Caprio, G., Dardano, P., Coppola, G., Cabrini, S., Mocella, V., "Digital holographic microscopy characterization of superdirective beam by metamaterial," Opt. Lett. 37(7), 1142 (2012).

[14] del Río, M. S., Dejus, R. J., "XOP v2.4: recent developments of the X-ray optics software toolkit," SPIE Optical Engineering + Applications 8141, M. Sanchez del Rio and O. Chubar, Eds., 814115-814115-5, International Society for Optics and Photonics (2011).

[15] Gronkowski, J., Malgrange, C., IUCr, "Propagation of X-ray beams in distorted crystals (Bragg case). I. The case of weak deformations," Acta Crystallogr A Found Crystallogr 40(5), 507-514, International Union of Crystallography (1984).

[16] Gronkowski, J., Malgrange, C., IUCr, "Propagation of X-ray beams in distorted crystals (Bragg case). II. The case of strong deformations," Acta Crystallogr A Found Crystallogr 40(5), 515-522, International Union of Crystallography (1984).

[17] Chukhovskii, F. N., Krisch, M., "The lens equation for Bragg diffraction optics. The general case of asymmetrical reflection," J. Appl. Cryst (1992). 25, 211-213 [doi:10.1107/S0021889891012074] 25(2), 1-3, International Union of Crystallography (1992). 